\title{
Multicultural care in nursing-From the theoretical paradigm to the subjective experiences in clinical settings
}

\author{
Ana Paula Teixeira de Almeida Monteiro ${ }^{1,2}$, Aida Cruz Mendes ${ }^{1,2}$ \\ ${ }^{1}$ Nursing School of Coimbra, Coimbra, Portugal \\ ${ }^{2}$ Unidade de Investigação em Ciências da Saúde-Enfermagem (UICISA-E), Coimbra, Portugal \\ Email: anapaula@esenfc.pt, acmmendes@esenfc.pt
}

Received 30 September 2013; revised 3 November 2013; accepted 15 November 2013

Copyright (C 2013 Ana Paula Teixeira de Almeida Monteiro, Aida Cruz Mendes. This is an open access article distributed under the Creative Commons Attribution License, which permits unrestricted use, distribution, and reproduction in any medium, provided the original work is properly cited.

\begin{abstract}
Last decade, the geography of immigration in Portugal experienced intense transformations with an increasing inflow of new and diversified migratory groups. An open questionnaire was developed and applied to 22 Portuguese nurses, aiming to analyse personal experiences and significant situations of nursing care in multicultural contexts. The understanding of hospital routines, difficulties in managing physical space due to different cultural standards, body exposure, and body handling in nursing clinical care, emerged as more significant issues in reports of critical incidents focusing on multicultural contexts.
\end{abstract}

Keywords: Multicultural Nursing; Mental Health and Culture

\section{INTRODUCTION}

During the last decade, the geography of immigration in Portugal experienced intense transformations in terms of recruitment and patterns of geographic settlement, with an increasing and exponential inflow of new and diversified migratory groups. The Portuguese health care system is still struggling to address some of the specific challenges presented by recent legal and illegal immigration from Brazil, Central and Eastern Europe and China, as well as more traditional immigration from Africa [1]. These populations have linguistic, cultural and sociodemographic specificities, which are very different from the general Portuguese population. This makes them a paradigmatic case in potential transcultural nursing studies.

For transcultural nursing, which integrated or systematized the contributions of Anthropology, studies on health problems in migrant populations and minority ethnic groups, the analysis of cultural variations in the subjec- tive experience of illness or the issue of cultural sensitivity of nursing care is particularly significant [2].

Transcultural nursing is an essential area of study and practice focused on the cultural care beliefs, values, and lifestyles of people to help them maintain and/or regain their health, or to face death in meaningful ways [3].

Essentially, transcultural nursing has focused on understanding cultures and their specific care needs and how to provide care that fits their lifestyles rather than assuming professional nurses always knowing what is best for them. The Process of Cultural Competence in the Delivery of Healthcare Services Model is a model of cultural competence that defines cultural competence as the process in which the nurse continuously strives to achieve the ability and availability to effectively work within the cultural context of a client individual, family or community. It includes consideration of cultural awareness, cultural knowledge, cultural skills and cultural encounters [4,5]. This model views cultural competence as the ongoing process in which the health care provider continuously strives to achieve the ability to effectively work within the cultural context of the client, individual, family, community. In Portugal this model is not commonly used in health units.

Cultural competency is a set of congruent behaviours, attitudes and policies that come together in a system, agency or among professionals that enable that system, agency or those professions to work effectively in crosscultural situations [6]. The range of definitions of crosscultural competence could be synthesized as the ability to function or work effectively in culturally diverse situations in general and in particular encounters with people from different cultures [7].

The systematized reflection of experiences lived in professional contexts may serve as anchorage for the cognitive reorganization and acquisition of skills in different areas of knowledge, particularly in acquiring and 
increasing a greater cultural sensitivity and competence for the practice of specialized nursing care. The objective of this pedagogic approach was to become acquainted with reality in a reflexive manner, share significant experiences, debate field trajectories or even disseminate innovative approaches to answer the common problems that Portuguese nurse's face in clinical practice in multicultural contexts. This approach, both experiential and cognitive, aimed at building interactive bridges between the existing knowledge of the students and new information and leading them toward improved cultural competence levels [8].

\subsection{Aim}

The objective of this research was to understand significant experiences and innovative approaches used to answer common problems faced by Portuguese nurses in multicultural contexts.

\subsection{Methods}

Data were gathered through open questionnaire, designed by the researcher, and answered by 22 nurses.

The nurses willingly agreed to answer the questionnaire in a pedagogic context. The study was conducted according to general ethical standards and qualitative study's protocols [9].

For ethical reasons and in order to ensure the complete anonymity and confidentiality of the data, there was no collection of detailed sociodemographic information regarding gender, age or marital status of the people involved in the cases reported, either nurses or patients. The objective was to analyse personal experiences of significant situations of confrontation with clinical experiences, in which the issues of multiculturalism of care and the expression of distress or symptoms were mediated by nurses.

The information gathered by questionnaire was also object of reflexive analysis in a pedagogic context (classroom), and a final qualitative analysis - a systematizing theme/category-based content analysis [10]—from which resulted this paper.

\subsection{Contexts}

The cases mentioned took place in several settings and clinical contexts: Surgical Units, Emergency Units, Psychiatric Units, Maternities and Operating Rooms. In terms of the ethnic groups involved, immigrant users of several nationalities stand out (Eastern Europe, Cape Verde, Japan, China and Cuba), but there were also very significant accounts of specific groups of the Portuguese population with differentiated cultural matrices, whose beliefs, values and life experiences have very particular shades, such as the elderly, the Roma people or people with religious beliefs of little expression in Portugal.

\section{RESULTS-EMERGING ISSUES}

These episodes of cultural divergence were lived and reported by nurses as critical incidents, sources of stress and perplexity, or even as potential conflicts in the management of clinical care. The qualitative analysis carried out allowed for the identification of some essential axes and central issues that will be presented in a systematic manner. Five main themes emerged from the data.

\subsection{Understanding and Accepting Hospital Routines/Managing the Physical Space}

A significant part of the cases reported have to do with emergent issues of confrontation/difficulty between caregivers and users as to understanding and accepting hospital routines (hygiene care, food, visits from relatives), managing the physical space and its symbolic meanings.

- Case 1-A patient that upon admission was not able to sleep in the bed. He removed the mattress, put it on the ground and only then would he lie down and fall asleep. We did not understand his behaviour, until we reached the conclusion that the patient had never slept in a bed and so he was accustomed to resting on the ground - he felt uneasy on the bed, he was afraid to fall down. We adapted the space and for a while we let the patient sleep as he wanted $\cdots$

- Case 2-A patient of Roma ethnicity refused to lie down in bed like the other patients and became very agitated. After listening to the patient we realized that it was because of the placement/orientation of the beds, he said "the dead are the ones who are laid down with their feet towards the door, ready to go to the cemetery" and to lie down like that in a hospital is a bad omen. This was the orientation of the beds in that unit and there was no way to change their position because of all the clinical equipment. So, we tried to adapt the space and the patient lay down in the bed and slept in a reversed position, with the head where the feet should be $\cdots$

\subsection{Explanatory Models of Illness and System of Care}

Another element of cultural divergence has to do with the explanatory models of illness and system of care. The various explanatory models of illness and healthcare diverge in terms of analytical skill, metaphors, and languages [11].

The cultural specificities of beliefs and subjective interpretations regarding the significance of symptoms or the etiological explanation of illnesses can collide with the parameters of nosological construction and therapeu- 
tic intervention of western medicine. This divergence is obvious in several ambivalent accounts regarding certain practices of "popular medicine" that continue to be expressed by patients and their families, even in health units with a high level of biomedical sophistication:

- Case 3-“( $\cdots)$ the family or someone close to the patient did not accept the illness and resorted to witchcraft and spiritualism to relief the suffering, although the person was hospitalized and receiving medical care...”

The western medical explanatory model differs greatly from other models that view illness more as an imbalance of forces or as being influenced by unseen forces such as spirits, demons or curses.

- Case 4- "The family of a patient in acute stage of schizophrenia visited him at the hospital and brought 'herbs' and 'holy waters', and performed some rituals, believing that would cure their relative. When the patient improved, he was very confused, since he did not know whether he was better, without symptoms, because of the rituals the family had performed or because of the medication...”

It is also in this context that we can explore other situations that are often mentioned-the refusal or difficulty in understanding certain technical interventions or more invasive technical procedures, interpreted and experienced with non-clinical connotations.

In one of the cases reported, the conscious refusal of the patient to comply with the therapeutic procedures (recommended by the health team) resulted in the death of the patient.

\subsection{Experiences in Immigration}

Generally Portuguese hospitals and health care centres have not developed specific practices for immigrants and there are no special facilities to which users can be referred. The language barrier as a fundamental obstacle was identified only by two of the nurses interviewed, while communication issues related with multicultural diversity emerged as relevant. Other issues identified were extreme solitude, lack of social support, feelings of hopelessness and difficulty in coping with family contexts. Within these cases, the most remarkable were those of people from other nationalities going through migratory processes or people belonging to minority ethnic groups.

- Case 5-“An Ukrainian youth that had been admitted in the emergency unit after having been run over was crying profusely, not just because of the pain, but also because he was far away from home, he felt mistreated and persecuted and because in the emergency unit all his clothes was cut off (at the time of the objective exam, for swift exposure of the body to look for injuries, which is usual with severely injured vic- tims). His Portuguese was reasonable and he said that he had nothing and no one to help him; he didn't even have another change of clothes. 'I just came looking for work and now I get killed'.”

\subsection{The Body Exposure}

The issue of exposure or manipulation of the body in the context of nursing clinical care emerged with some intensity in accounts of critical incidents, in which the cultural interpretations clearly related to boundaries of care were central.

Mass clinical practice and health technologies in a hospital context with highly invasive and specialized procedures, tend not to give priority to psychological or emotional aspects like modesty, sense of privacy, fear or boundaries of the self through the control of ones' own body. When a person enters a hospital or a nursing home, the body becomes a terrain of medical practices and care. This implies that the body is an object external to the self and will be subjected to the authority of others: physicians and nurses. This mechanism allows people to touch and to be touched in a way which is considered inappropriate and a matter out of place in another context. The private space of the body is altered and boundaries of the body are redefined. In the healing process, some of the most important (sometimes implicit) rules regulating interpersonal contact in the outside world simply disappear $[12,13]$. However touching and body exposure in clinical contexts also reflects specific cultural ideas, values and norms in a society. The way nurses deal with the exposure and manipulation of the body in a context of clinical care has been the subject of some anthropological approaches that highlight some structural ambivalence. Touch in nursing has a double meaning: on the one hand, it is a utilitarian and technical activity in which the bodies of nurses and patients are objects. In hospitals, direct touch is often replaced with technological artefacts', as disposable gloves used during medical examinations and procedures, hemodynamic monitoring electronic systems (to control arterial blood pressure, temperature or heart rate and all the electronic devices), and all the other devices that "sterilize" or make human contact skin to skin useless. This means that a nurse can touch the body-object without feeling emotional involvement. The body-object is a "thing" to be cared for. On the other hand, the patient's body is never just a thing for nurses; it shows reactions and emotions, it responds to touch and interpersonal relationship [14].

- Case 6- "Regarding the exposure of the body, there are patients for whom the exposure of parts of the body, like the head, is particularly unacceptable, thus resulting in almost ridicule situations. An extreme situation was that of an elderly woman belonging to a religious congregation; we were doing the pre-op- 
eratory preparation and when we were about to send her to the Operating Room, we found her with her head completely covered with a pillowcase, as if it were a veil, and the entire body wrapped in bed covers."

The perplexity of nurses results from a cultural divergence-for a woman marked by religious seclusion, where the veil has a strong symbolism and cultural identity, it was an act of somewhat institutional revolt regaining the original form of the protective veil rather than the asepsis of the surgical protocol.

\subsection{The Difficulty in Diagnosing in Psychiatry}

Another analytical category that emerged was the difficulty in establishing clinical diagnoses in contexts of cultural diversity, particularly in psychiatric units. The critical analysis of the categories of diagnosis of western classical psychiatry applied to different cultures has led to a focus on the difficulties of comparative studies of culturally differentiated ethnic groups. Diagnosis across cultural boundaries has become a practical rather than an esoteric matter as migration, the number of effective psychiatric therapies, and access to psychiatric care have increased. Cross-cultural diagnosis involves such theoretical considerations as diagnostic categories, pathoplasticity of psychiatric disorder, culture-bound syndromes, "emic” “(intracultural)” versus “etic” (cross-cultural) conceptual frameworks, and different reporting of symptoms and expression of signs from one cultural group to another [15].

Explanatory models of mental illness are likely to vary from culture to culture, depending on beliefs about normative development and the relative value of different behaviours in a culture. Every culture has a rich set of idioms for the expression of distress aimed at mobilizing an effective social response [16,17]. From the perspective of cultural psychiatry, culture influences the sources, the symptoms and the idioms of distress; the individuals' explanatory models, their coping mechanisms and their help-seeking behaviour; as well as the social response to distress and to disability [18].

The prevailing approach considers that the diagnostic categories of western classical psychiatry are universally valid as nosological categories, despite some local variations in terms of the expression of symptoms. Subjacent to that is an organizational and biological perspective of mental illness which, although shaped and influenced by social-cultural contexts, has a biological expression that is independent of cultural diversity. However, some theoreticians consider that the cultural validity of psychiatric diagnoses, applied to specific populations, implies that the nosological criteria need to be redefined or even restructured according to the several cultural matrixes analysed. There is always the possibility of having a con- ceptual and analytical deviation when a determined pathology is classified/diagnosed in a determined cultural context and is based on diagnostic categories produced by another culture, thus leading to a fallacy of category [19]. Nurses in contexts of clinical practice in psychiatric units tend to integrate that dominant biomedical model. However, the difficulties in interpreting the seemingly dysfunctional behaviour of patients do emerge and are the subject of critical examination:

- Case 7-“An immigrant woman from a completely different culture, with almost no shared characteristics, was hospitalized after being diagnosed with a 'psychotic break'. She had very bizarre behavior and had no knowledge of the Portuguese language... As time went by, with more up-close and direct nursing care, having contact with relatives of the same nationality, we realized that we were very unskilled in the analysis of her behavior in light of her culture and that the symptoms presented had not been well understood... Probably the patient had not been 'well diagnosed' and the changes she presented were not psychotic in any way...”

\section{DISCUSSION: BUILDING BRIDGES IN NURSING CARE}

In Portugal, the training of psychiatric and mental health nurses consists of a four-year-long broad undergraduate degree, followed by a two-year specialized graduate degree. This means that psychiatric and mental health nurses work in diverse health units, both in hospitals and primary care. This experience is innovative since it allows the development of mental health care within other medical specialties. This study reveals how mental health units show greater sensitivity to multicultural issues in comparison to other nursing units. Mental health nurses who are placed in multi-specialty teams can contribute to a larger promotion of culturally sensitive health care across the entire health system. This study also highlights a few key issues that are important for mental health and psychiatric nurses such as space-organization, therapeutic environment management, and culturally distinctive ways of communication.

Through the analysis of the narratives we may see that the nurses involved in critical incidents, and the health teams to which they belong, use, in their daily practice, types of cultural sensitivity that are not always structured, using tools of negotiation, flexibility or even creativity in the management of the difficulties of cultural divergence. However, this organizational flexibility is strongly mediated by the clinical settings in which it takes place-it tends to be broader when the care is not carried out in more technologically sophisticated contexts like Emergency Units, Operating Rooms and Surgery. In other words, flexibility and respect for cultural specificity in 
care are more visible when clinical interventions are seen as accessories (and therefore negotiable), as in situations in which there is no immediate life risk for the patient. Such is the case of psychiatry and maternity services. There is something else that emerges from the analysis of cases: nursing theories (based on personalized care and the holistic approach to the human being) tend to integrate multicultural diversity and promote greater sensitivity and congruence in healthcare, with some tension and ambivalence regarding the dominant biomedical model, based on intensive biotechnologies, which are typical of highly specialized health units. In psychiatric units there appears to be a greater perception of the intrinsic cultural variations in the processes of illness and emotional suffering.

In addition, this study reveals the difficulty in making neutral psychiatric diagnosis. The cultural content of psychiatric diagnosis should include the main, well-recognized cultural variables, adequate family data, explanatory models, and strengths and weaknesses of every individual patient [20]. Gaining a better understanding of someone else's explanatory model will not in and by itself resolve conflicts in mental health nursing care. However, a foundation can be established for negotiating a course of care that is acceptable within both the Western medical model and the model of the patient and family. Negotiation and compromise are critical [21].

\section{CONCLUSIONS}

The training of mental health nurses should include a strong awareness of culture-bound syndromes (several kinds of illness or affliction), all of which are defined but not having a one-to-one correspondence with a disorder recognized by Western, allopathic nosologies.

The issue of culturally sensitive nursing care also entails a structural and organizational change in healthcare services and units, including psychiatric units. The models of clinical assistance and mass clinical practice in hospital contexts are generally characterized by patterns of organizational efficacy that involve highly invasive and specialized procedures, as well as being technologically standardized. From this perspective, cultural dimensions do not tend to be prioritized in face of indicators of profitability and clinical efficacy that condition nursing interventions. Nonetheless, clinical practice is confronted with the need to see to psychological or emotional aspects like decency, sense of privacy, fear, intense anxiety or solitude. The experiences of care in clinical contexts that were reported showed that culture has a strong influence on experience and the social representation of illness, as well as in the way symptoms are experienced, expressed and interpreted, both subjectively and socially.

The study carried out seems to strengthen the need to implement in Portugal Programs of Intercultural Mediation in the network of hospitals and healthcare centres, with referenced translators, specially trained to approach extremely sensitive areas like mental health or the support to families during periods of transition. In Portugal, only a few pilot-projects are using Intercultural Mediation Program in health. The aims of the intercultural mediation are to improve access and the quality of health care delivered to ethnic minority patients at the hospital, and also to improve communication and responsiveness to the socio-cultural and health-care needs of ethnic minority patients. An Intercultural Mediator usually shares the cultural background of the patient, and commonly takes on the tasks of interpretation, cultural decoding, and accompanying patients in the hospital, but these projects are very scarce in Portugal, and health professionals face difficulty in understanding their patients in daily hospital routines.

With the rapid changes and influx of new populations from around the world, nurses are, more than ever before, faced with the challenge of caring for many different cultural groups. Changes are occurring more rapidly in urban, suburban, and rural areas, often with cultural groups clustering together in ethnic neighbourhoods. This means that there is less time for nurses to learn about and become accustomed to new cultural groups [22].

Also the results of this study underline the need for continuous training programs for health professionals. The implementation of this core curriculum in multicultural care should be longitudinal. Such strategy should facilitate a better understanding of various cultural methods for prevention, healing, and caring, promoting a culturally sensitive healthcare practice [23].

Cross-cultural training is a complex and long-term process. Information components are generally well-received and can be developed over a relatively short period of time but changing attitudes and sensitivities requires gradual and progressive engagement [24,25]. Training programs should be based on adult learning principles and include a variety of approaches and should favour more experiential approaches [7].

\section{REFERENCES}

[1] Caldas-de-Almeida M.B., Funkm, M. and Muijen M. (2009) Portugal WHO country summary. http://www.acs.min-saude.pt/files/2010/03/Portugal-Coun try Summary_FINAL_para_WEB.pdf

[2] Monteiro A. (2005) Multiculturalismo e cuidados de enfermagem. Pensar Enfermagem, 9, 48-57.

[3] Leininger, M. (1999) What is transcultural nursing and culturally competent care? Journal of Transcultural Nursing, 10, 9.

http://dx.doi.org/10.1177/104365969901000105 
[4] Campinha-Bacote, J. (1999) A model and instrument for addressing cultural competence in health care. Journal of Nursing Education, 38, 203-207.

[5] Campinha-Bacote, J. (2003) The process of cultural competence in the delivery of healthcare services: A culturally competent model of care. 4th Edition, Transcultural C.A.R.E. Associates, Cincinnati.

[6] Cross, T., Bazron, B., Dennis, K. and Isacs, M. (1989) Towards a culturally competent system of care. Georgetown University, Washington DC.

[7] Bean, R. (2006) Cross-cultural competence and training in Australia. Cultural competency in the healthcare industry.

http://www.ofv.sa.gov.au/pdfs/CONGRESS_2007_Cross CulturalTrainingPaper_RBean.pdf

[8] Hunter, L. (2009) Applying constructivism to nursing education in cultural competence-A course that bears repeating. Journal of Transcultural Nursing, 19, 354-362. http://dx.doi.org/10.1177/1043659608322421

[9] Beauchamp, T.L. and Childress, F. (2001) Principles of biomedical ethics. 5th Edition, Oxford University Press, Oxford.

[10] Bardin, L. (1977) L’analyse de contenu. Presses Universitaires de France, Paris.

[11] Kleinman, A. (1980) Patients and healers in the context of culture: an exploration of the borderland between anthropology, medicine, and psychiatry. University of California Press, Berkeley.

[12] Picco, E., Santoro, R. and Garrino, L. (2010) Dealing with the patient's body in nursing: Nurses' ambiguous experience in clinical practice. Nursing Inquiry, 17, 3946. http://dx.doi.org/10.1111/j.1440-1800.2009.00481.x

[13] Le Breton, V. (2011) Images culturelles du corps. Entre organisme et chair. Medicine/ Sciences, 27, 311-314.

[14] Van Dongen, E. and Elema, R. (2001) The art of touching: The culture of "body work" in nursing. Anthropology \& Medicine, 8, 149-162. http://dx.doi.org/10.1080/13648470120101345
[15] Westermeyer, J. (1985) Psychiatric diagnosis across cultural boundaries. American Journal of Psychiatry, 142, 798-805.

[16] Kirmayer, L. (2004) The cultural diversity of healing: Meaning, metaphor and mechanism. British Medical Bulletin, 69, 33-48. http://dx.doi.org/10.1093/bmb/ldh006

[17] Kirmayer, L. (2005) Culture, context and experience in psychiatric diagnosis. Psychopathology, 38, 192-196. http://dx.doi.org/10.1159/000086090

[18] Bhugra, D. and Mastrogianni, A. (2004) Globalisation and mental disorders overview with relation to depression. The British Journal of Psychiatry, 184, 10-20. http://dx.doi.org/10.1192/bjp.184.1.10

[19] Kleinman, A. (1988) The illness narratives: Suffering, healing, and the human condition. Basic Books, New York.

[20] Alarcón, R. (2009) Culture, cultural factors and psychiatric diagnosis: Review and projections. World Psychiatry, 8, 131-139.

[21] Hallenbeck, J. (2002) Cross-cultural issues. In: Berger, A., Portenoy, R.K. and Weissman, D.E., Eds., Principles and Practice of Palliative Care and Supportive Oncology, 2nd Edition, Lippincott Williams \& Wilkins, Philadelphia, 661-672.

[22] Jeffreys, M. (2010) Teaching cultural competence in nursing and health care. 2nd Edition, Springer Publishing Company, New York.

[23] Monteiro, A.P. (2011) Migraçã̃o e saúde mental. Psicosoma, Viseu.

[24] Graf, A. (2004) Assessing intercultural training designs. Journal of European Industrial Training, 28, 199-214. http://dx.doi.org/10.1108/03090590410527618

[25] Chrisman, N.J. (2007) Extending cultural competence through systems change: Academic, hospital, and community partnerships. Journal of Transcultural Nursing, 18, 68S-76S. http://dx.doi.org/10.1177/1043659606295692 